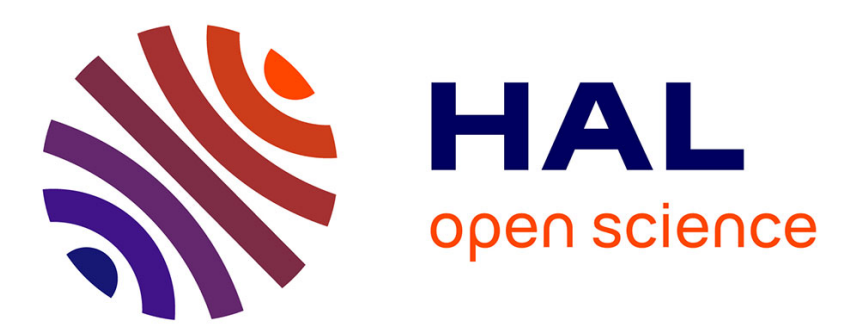

\title{
Robustness of structural reliability analyses to epistemic uncertainties
}

\author{
Mohamed Guedri, Scott Cogan, Noureddine Bouhaddi
}

\section{To cite this version:}

Mohamed Guedri, Scott Cogan, Noureddine Bouhaddi. Robustness of structural reliability analyses to epistemic uncertainties. Mechanical Systems and Signal Processing, 2012, 28, pp.458-459. 10.1016/j.ymssp.2011.11.024 . hal-02300226

\section{HAL Id: hal-02300226 \\ https://hal.science/hal-02300226}

Submitted on 20 Nov 2019

HAL is a multi-disciplinary open access archive for the deposit and dissemination of scientific research documents, whether they are published or not. The documents may come from teaching and research institutions in France or abroad, or from public or private research centers.
L'archive ouverte pluridisciplinaire HAL, est destinée au dépôt et à la diffusion de documents scientifiques de niveau recherche, publiés ou non, émanant des établissements d'enseignement et de recherche français ou étrangers, des laboratoires publics ou privés. 


\title{
Robustness of structural reliability analyses to epistemic uncertainties
}

\author{
M. Guedri ${ }^{\text {a,*}}$, S. Cogan ${ }^{\text {b }}$, N. Bouhaddi ${ }^{b}$ \\ a Preparatory Engineering Institute of Nabeul (IPEIN), 8000 M'rezgua, Nabeul, Tunisia \\ ${ }^{\mathrm{b}}$ FEMTO-ST Institute UMR 6174 - Applied Mechanics Laboratory R. Chaléat, University of Franche-Comté, 24 Chemin de l'Epitaphe, 25000 Besançon, France
}

\begin{abstract}
Methods for the robust design of mechanical systems have the objective to reduce the variability in system performance with respect to uncertainties in the material and geometrical properties of a mechanical structure as well as in its interactions with its environment. Two types of uncertainty are encountered in practice, namely aleatory and epistemic uncertainties. Aleatory uncertainty is generally considered to be irreducible and results from statistical variations in the physical properties of components and interfaces. Epistemic uncertainties are due to a lack of accurate knowledge concerning the physical laws governing the behavior of a component or interface and can generally be reduced with a combination of more detailed modeling and experimental investigations. Epistemic uncertainties can be difficult to characterize due to simplifications in geometric and material field properties, and as such are rarely taken into account explicitly in reliability analysis. In the present work, we propose to examine the robustness of a classical reliability analysis with respect to both aleatory and epistemic uncertainty. The latter will be represented using a non-parametric approach in order to avoid a detailed characterization of the lack of knowledge present in the system. This then allows us to study in detail how the results of the reliability analysis vary as a function of the degree of lack of knowledge. The proposed methodology is illustrated using numerical simulations.
\end{abstract}

\section{Introduction}

In engineering problems, uncertainty is omnipresent and the scatter of parameters from their nominal values is unavoidable. Uncertainties in structural mechanics, and in particular during the early phases of analysis and design, can play an extremely important role, affecting not only the safety and reliability of structures and their mechanical components, but also the level of their performance. Indeed, the response of a structural system can be very sensitive to uncertainties in the material properties, manufacturing conditions, external loading and analytical or numerical modeling. Stochastic analysis methods have been developed over the last decades in order to account for some forms of uncertainty encountered in structural mechanics [1].

The development by the scientific community of probabilistic analysis methods over the last two decades has stimulated the interest for considering uncertainty in the formulation of the structural design optimization problem $[2,3]$.

\footnotetext{
* Corresponding author. Tel.: +216 72220 053; fax: +216 72220181 .

E-mail address: mohamed.guedri@isetn.rnu.tn (M. Guedri).
} 
In order to account for uncertainties in this framework, probability-based formulations must be implemented via stochastic simulations. The probability-based design optimization methodologies can be broadly classified in the following three categories [4]: (i) Robust Design Optimization (RDO); (ii) ReliabilityBased Design Optimization (RBDO) and (iii) Reliabilitybased Robust Design Optimization (RRDO).

Generally, the uncertainties can be categorized as: (i) aleatory uncertainty (variability, irreducible, random, inherent or stochastic) and (ii) epistemic uncertainty (reducible, subjective, state-of-knowledge, model form or simply uncertainty). Recently Oberkampf et al. [5] and He et al. [6] added the notion of error (numerical uncertainty), which is generally placed in (ii). Aleatory uncertainty can be characterized by known probability distributions while epistemic uncertainty arises from a lack of knowledge in either probabilistic or non-probabilistic quantities.

The distinction between these two types of uncertainty can be useful in engineering analysis because epistemic uncertainty is reducible. Although some have suggested that a clear distinction between the two types can be made, in the modeling phase it is often difficult to determine whether a particular uncertainty should be put into the aleatory or the epistemic category, and thus the distinction is mainly determined by our modeling choices [7]. In many cases, both aleatory and epistemic uncertainty can be treated and analyzed, either separately or combined, using probability theory and statistics $[8-10]$.

The sources of aleatory uncertainty are typically represented using a probabilistic framework based on a finite number of random variables with some known distribution. These may include both uncertainties in model coefficients (parametric uncertainty) as well as uncertainty in the sequence of possible events (stochastic uncertainty). Stochastic uncertainty is entirely aleatory by nature. Parametric uncertainty can also be completely aleatory if the complete distributions of all the model parameters are known a priori (probabilistic distribution) [11]. Aleatory uncertainty has been studied extensively within classical information theory with the principle of maximum entropy, which was presumably founded by Jaynes [12]. The book by Kapur [13] is an excellent overview of the wide range of applications of this principle.

Epistemic uncertainties are related to the lack of information that could in principle be reduced by additional information. This type of uncertainty includes model errors and errors introduced by the numerical solution of the corresponding problem. In certain cases of distributed uncertainty due to geometric and material simplifications, the parametric approach is not suitable and a non-parametric approach [14-16] is needed for this purpose.

In the field of uncertainty quantification, extensive research has been devoted to the numerical treatment of aleatory uncertainty $[17,18]$. Meanwhile, little attention has been given to the quantification of epistemic uncertainty. Adhikari proposed a non-parametric approach based on a new optimal Wishart distribution for the quantification of epistemic uncertainty [15,16]. Recently, Jakeman et al. [11] also proposed a numerical framework for the quantification of epistemic uncertainty.

Uncertainties in structural mechanics, analysis and design play an extremely important role. They affect the safety and reliability of structures and mechanical components. The following paragraphs examine the influence of the two types of uncertainties (aleatory and epistemic uncertainties) in reliability assessment.

The non-probabilistic methods (which belongs to epistemic uncertainties) for analyzing reliability have been brought forward by Ben-Haim and Elishakoff [19-22] and Au et al. [23]. Elishakoff proposed the notion of the non-probabilistic safety factor, which is defined as the ratio of the yield stress - in case it is a deterministic quantity - by the upper bound of stress. Ben-Haim proposed the concept of the non-probabilistic reliability of structures, called info-gap robust reliability.

Basically, the uncertainty models employed by a typical structural reliability analysis can be classified into two categories: the probabilistic model and non-probabilistic models. When probabilistic and non-probabilistic variables appear in the same problem, the failure probability can be provided by the hybrid reliability model [24,25]. A combination of stochastic variables and uncertain-but-bounded variables has been suggested for applications in such circumstances [26,27]. Some numerical methods, including the function approximation technique [28], the iterative rescaling method [29] and the probability bounds (p-box) approach [30], have been proposed for the estimation of lower and upper bounds of the structural reliability in the presence of both stochastic and interval variables. For a detailed review on the methods, see $[24,25]$.

The reliability assessment of structures exhibiting both stochastic and bounded uncertainties using a probability and convex set mixed model was proposed by Luo et al. [24], and the calculation of the corresponding reliability index was formulated mathematically as a constrained minimization problem. Based on the probabilistic and multi-ellipsoid convex set hybrid model, Zhan and Luo [31] presented a mathematical definition of the reliability index for measuring the safety of structures in presence of parameters or load uncertainties. The optimization problem was reformulated into a more tractable form using the performance measure approach, and to further facilitate the efficient solution of the design problem, the nested double-loop optimization problem was transformed into an approximate single-loop minimization problem.

Recently, Ni and Qiu proposed a new hybrid reliability model, which contains randomness, fuzziness and nonprobabilistic uncertainty based on the structural fuzzy random reliability and non-probabilistic set-based models [25] based on the conventional probabilistic model [32] and non-probabilistic set-based model [33]. This new hybrid model has broad applicability, which can handle either linear or non-linear state functions.

In the recent work [34], Rabhi et al. proposed an approach combining a dynamic reliability method and a meta-model (reduced model) to obtain good results in terms of the reliability and optimization of such systems. This method uses the hybrid model coupling of the possibility and probability approaches for the propagation of the uncertainties in the model. 
In this paper we propose to examine the robustness of a classical reliability analysis with respect to aleatory and epistemic uncertainty. In Section 2, the general concepts of aleatory and epistemic uncertainties in the context of reliability are presented. The aleatory uncertainty and the historical background of the random matrix distributions and applications of random matrix theory are mentioned. In Section 3, the dynamical system with model uncertainty is represented using the non-parametric probabilistic approach. Two computational procedures, the Monte Carlo Simulation (MCS) method and Importance Sampling (IS), are used in Section 4 to evaluate the failure probability. The proposed methodology is then illustrated by numerical examples in Section 5.

\section{General concepts of aleatory and epistemic uncertainties in the context of reliability}

\subsection{Aleatory uncertainty}

In reliability analysis, the performance of a structure is governed by a state function of a vector of basic random variables (RVs). The probability of failure is expressed as the integral of the joint probability density function over the failure domain. Let the random vector be denoted by $X=\left(X_{1}, X_{2}, \cdots, X_{n}\right)$; where $n$ is the number of basic RVs and the joint probability density function by $f_{X}(x)$; then the failure probability is expressed by

$$
P_{f}=P_{r}[g(x) \leq 0]=\int_{g(x) \leq 0} f_{X}(x) d x
$$

where $P_{r}[\bullet]$ denotes the probability and $g(x)$ is the state function defined as follows:

$$
\begin{aligned}
& g(x)=0: \text { the limit state; } g(x)>0 \text { : the safe domain } \\
& g(x) \leq 0 \text { : the failure domain }
\end{aligned}
$$

In practice $g(x)$ may or may not be explicit, and the analytical solution of Eq. (1) is in many cases difficult to obtain. Therefore, various methods and algorithms have been proposed to approach this integration.

Over the past three decades, there has been extensive research, e.g. Thoft-Christensen and Baker [35]; Ditlevsen and Madsen [32], to develop approximate numerical methods for the efficient calculation of the reliability integral. The approximate reliability methods can be broadly grouped into (i) first order reliability method (FORM) and (ii) second order reliability method (SORM). In FORM and SORM, it is assumed that all the basic random variables are transformed and scaled so that they are uncorrelated Gaussian random variables, each with zero mean and unit standard deviation.

In the case that the state function is not explicitly known, FORM/SORM are not directly applicable and a Monte Carlo simulation (MCS) is generally implemented. MCS can be used for analyzing uncertainty propagation, where the goal is to determine how random variation, lack of knowledge, or error affects the sensitivity, performance or reliability of the system that is being modeled. It is categorized as a sampling method because the inputs are randomly generated from probability distributions to simulate the process of sampling from an actual population. The choice of distribution for the inputs should closely match the available data, or best represent the current state of knowledge. The MCS technique has the important property that the successive points in the sample are independent [1].

The main advantages of MCS method [36,1] are: (i) the capability of handling practically any mechanical or physical model regardless of its complexity; and (ii) its simple implementation without any modification of the mechanical model, which can be considered as a "black box" receiving simple analysis calls. The main disadvantages are: (i) the excessive computational effort due to the large required sample size, especially for realistic structures with low probability of failure; and (ii) the numerical noise due to random sampling, leading to nonmonotonic estimates during simulations, and as a result, it becomes impossible to obtain an accurate and stable evaluation of the response gradient.

To overcome these disadvantages, numerous variance reduction techniques have been proposed, including conditional expectation (CE) [37], response surface approximation (RSM) [38], Latin hypercube sampling (LHS) [39], importance sampling (IS) [40-42] and directional simulation (DS) $[43,44]$.

\subsection{Epistemic uncertainty}

Epistemic uncertainty is due to the lack of knowledge and generally arises in the modeling of complex systems. Due to its very nature, it can be comparatively difficult to quantify or model epistemic uncertainty. In the present context, a nonparametric approach is proposed.

The interested reader can refer to the works of Adhikari [15,16] for detailed historical background on random matrix distributions and applications of random matrix theory. According to Adhikari [15,16], the following probability density functions are relevant to stochastic mechanics problems: (i) Gaussian random matrix: Gaussian random matrix is constituted of real random matrix whose elements are independent and identically distributed standard normal random variables [45]. (ii) Wishart matrix: An optimal Wishart distribution has been proposed by Adhikari [15] to model random system matrices. The probability density functions of the system matrices are derived using the maximum entropy method. (iii) Matrix variate gamma distribution: The matrix variate gamma distribution has been used by Soize [14,46,47] for the random system matrices of linear dynamical systems. (iv) Noncentral Wishart matrix: The Noncentral Wishart 
distribution has been used by Adhikari [16] to build a unified approach to model simultaneous parametric and nonparametric uncertainties.

In the present work, we propose to use the non-parametric approach proposed by Soize $[14,47]$ in order to introduce the level of epistemic uncertainty.

\section{Dynamical system with model uncertainty represented by the non-parametric probabilistic approach}

The equation of motion of a damped n-degree-of-freedom linear dynamical system can be expressed as:

$$
\mathbf{M} \ddot{\mathbf{u}}(t)+\mathbf{B} \dot{\mathbf{u}}(t)+\mathbf{K u}(t)=\mathbf{f}(t)
$$

where $\mathbf{M} \in \mathfrak{R}^{n \times n}, \mathbf{B} \in \mathfrak{R}^{n \times n}$ and $\mathbf{K} \in \mathfrak{R}^{n \times n}$ are the stochastic mass, damping and stiffness matrices, respectively.

In this model the $\mathbf{M}, \mathbf{B}$ and $\mathbf{K}$ are assumed to be random matrices. The distribution of these random matrices should be such that they are: (a) symmetric, (b) positive-definiteand (c) the moments of the inverse of the dynamic stiffness matrix:

$$
\mathbf{D}(\omega)=-\omega^{2} \mathbf{M}+i \omega \mathbf{B}+\mathbf{K}
$$

should exist $\forall \omega$. That is, if $\mathbf{H}(\omega)$ is the frequency response function (FRF) matrix:

$$
\mathbf{H}(\omega)=\mathbf{D}^{-1}(\omega)=\left[-\omega^{2} \mathbf{M}+i \omega \mathbf{B}+\mathbf{K}\right]^{-1}
$$

then the following condition must be satisfied for positive values of $v$ :

$$
E\left[\|\mathbf{H}(\omega)\|_{F}^{v}\right]<\infty, \quad \forall \omega
$$

where $E(\bullet)$ is the mathematical expectation operator.

The formal probabilistic description of the random positive-definite symmetric real matrix $\mathbf{G}$ is described in $[14,47]$. For numerical calculations, i.e. Monte Carlo simulation, the following procedure has been proposed to generate realizations of the random matrix G:

$$
\mathbf{G}=\mathbf{L}_{n}^{T} \mathbf{L}_{n}
$$

In the above equation $\mathbf{L}_{n} \in \mathfrak{R}^{n \times n}$ is an upper triangular random matrix resulting from the Cholesky factorization. The random variables of $\mathbf{L}_{n}$ are defined by a positive-valued gamma random variable [14,47].

The scalar $\delta$ is known as the dispersion parameter that characterizes the degree of uncertainty in the random matrix $\mathbf{G}$. The parameter $\delta$ is defined by

$$
\delta=\left\{\frac{E\left[\|\mathbf{G}-E[\mathbf{G}]\|_{F}^{2}\right]}{\|E[\mathbf{G}]\|_{F}^{2}}\right\}^{1 / 2}=\left\{\frac{1}{n} E\left[\left\|\mathbf{G}-\mathbf{I}_{n}\right\|_{F}^{2}\right]\right\}^{1 / 2}
$$

and it allows the dispersion of the probability model of random matrix $\mathbf{G}$ to be defined. In Ref. [46], it is shown that the dispersion of the probability model is set by specifying the parameter $\delta$, which must be independent of $n$ and such that

$$
0<\delta<\sqrt{(n+1) /(n+5)}
$$

The nonparametric random matrices $\mathbf{M}, \mathbf{B}$ and $\mathbf{K}$ must be positive definite and independent. It follows then that:

$$
\begin{aligned}
& E\{\mathbf{M}\}=\underline{M} ; \quad E\{\mathbf{B}\}=\underline{B} ; \quad E\{\mathbf{K}\}=\underline{K} \\
& E\left\{\left\|\mathbf{M}^{-1}\right\|_{F}^{2}\right\}<+\infty ; \quad E\left\{\left\|\mathbf{B}^{-1}\right\|_{F}^{2}\right\}<+\infty ; \quad E\left\{\left\|\mathbf{K}^{-1}\right\|_{F}^{2}\right\}<+\infty
\end{aligned}
$$

where $M, \underline{B}$ and $\underline{K}$ are the mean mass, damping and stiffness matrices, respectively. These matrices can then be written in the following form:

$$
\underline{M}=\underline{L}_{M}^{T} \underline{L}_{M} ; \quad \underline{B}=\underline{L}_{B}^{T} \underline{L}_{B} ; \quad \underline{K}=\underline{L}_{K}^{T} \underline{L}_{K}
$$

or again:

$$
\mathbf{M}=\underline{L}_{M}^{T} \mathbf{G}_{M} \underline{L}_{M} ; \quad \mathbf{B}=\underline{L}_{B}^{T} \mathbf{G}_{B} \underline{L}_{B} ; \quad \mathbf{K}=\underline{L}_{K}^{T} \mathbf{G}_{K} \underline{L}_{K}
$$

where $\mathbf{G}_{M}, \mathbf{G}_{B}$ and $\mathbf{G}_{K}$ are the random matrices defined by Eq. (7). The parameters $\delta_{M}, \delta_{B}$ and $\delta_{K}$ allow the dispersion of random matrices $\mathbf{M}, \mathbf{B}$ and $\mathbf{K}$ to be independently controlled are defined by Eq. (8) and must satisfy Eq. (9).

\section{Reliability analysis under aleatory and epistemic uncertainty}

In this section, two computational procedures, the Monte Carlo Simulation (MCS) method and Importance Sampling (IS), are presented for evaluating the failure probability. 


\subsection{Direct Monte-Carlo simulation techniques}

MCS methods [48] are a class of computational algorithms that rely on repeated random sampling to compute their results and are often used for simulating physical and mathematical systems. MCS offers the most robust method for calculating the probability of failure $P_{f}$ defined in Eq. (1) (or its complement to 1, the reliability). The estimate $P_{f}$ obtained through MCS is obtained easily as follows:

$$
P_{f} \approx \frac{N_{f}}{N}
$$

where $N$ and $N_{f}$ are the total number of simulated samples and number of failure samples, respectively. This estimate of $P_{f}$ is unbiased and its accuracy does not depend on the geometry of the failure domain or on the number $M$ of the random parameters involved. Instead, it only depends on $P_{f}$ and the number of samples $N$ used in the simulation. It is recommended to measure the statistical accuracy of the estimated probability of failure by computing its coefficient of variation (COV). The COV of $P_{f}$ is given by

$$
\operatorname{cov}\left(P_{f}\right)=\sqrt{\frac{1-P_{f}}{N P_{f}}}
$$

The smaller the coefficient of variation, the better the accuracy of the estimated probability of failure. For a small probability of failure and a small number of simulation cycles, the variance of $P_{f}$ can be quite large. Consequently, it may take a large number of simulation cycles to achieve a specific accuracy [49].

Variance reduction techniques offer an increase in the efficiency and the accuracy of the failure probability assessment in comparison with Direct Monte-Carlo simulation, for the same number of runs. Several variance reduction techniques exist: conditional expectation (CE) [37], response surface approximation (RSM) [38], Latin hypercube sampling (LHS) [39], importance sampling (IS) [40-42], directional simulation (DS) [43,44], etc. We present in the following the Importance sampling technique, which will be used in the numerical simulations.

\subsection{Importance sampling}

Importance Sampling is a general technique for estimating the properties of a particular distribution, while only having samples generated from a different distribution rather than the distribution of interest. The main idea behind the method is that certain values of the input random variables in a simulation have more impact on the parameter being estimated than others. If these "important" values are emphasized by sampling more frequently, then the estimator variance can be reduced. The basic methodology is to choose a distribution, which "encourages" the important values.

Importance Sampling consists in biasing the simulation by the use of another distribution so that the part of the integrand in Eq. (1) that makes the largest contribution is emphasized in the sampling (Fig. 1). To avoid introducing a bias in the final result, the estimation is corrected at the end.

The failure probability is then expressed as

$$
P_{f} \approx \frac{1}{N} \sum_{i=1}^{N} I_{f} \frac{f_{X}\left(x_{1 i}, \ldots, x_{n i}\right)}{h_{X}\left(x_{1 i}, \ldots, x_{n i}\right)}
$$

where $N$ is the number of simulations, $f_{X}\left(x_{1 i}, \ldots, x_{n i}\right)$ is the original joint density function and $h_{X}\left(x_{1 i}, \ldots, x_{n i}\right)$ is the importance density function and $I_{f}$ is the failure indicator function that takes values of 0 for failure or 1 for survival.

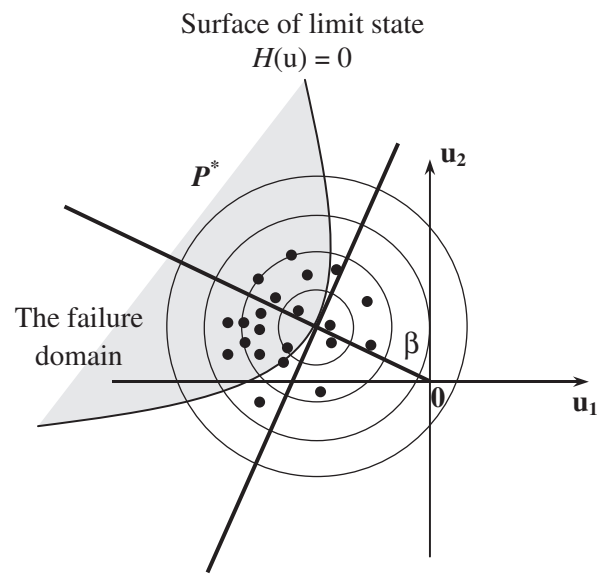

Fig. 1. Illustration of the importance sampling. 
The efficiency of this method depends on the choice of the biased distribution, which will encourage the important regions of the input variables. A good distribution can significantly reduce the computational time, in particular when estimating rare event probabilities.

\section{Numerical simulations}

\subsection{A ten DOF system}

This example was studied by Jensen and Valdebenito using a parametric reliability approach in the benchmark study [50]. We will study the same example, consisting in a damped linear array of spring-mass oscillators, to illustrate the proposed methodology and compare its performance with existing methods. The test case is a ten-degree-of-freedom oscillator with uncertainties appearing in the material parameters and the time history of the base excitation. The system, together with the numerical values assumed for different parameters, is shown in Fig. 2.

The equation of motion of a system with $n$ degrees of freedom submitted to a base excitation is analogous to that of a single degree of freedom system [51]. Consider the mass-spring system shown in Fig. 2 where

- $u_{g}(t)$ the displacement of the base

- $u_{i}^{t}$ the absolute displacement of each mass $m_{i}$ in the fixed reference $\left(x_{0}, y_{0}\right)$

- $u_{i}$ the relative displacement of these mass compared with the structure base.

The displacements $u_{g}, u_{i}$ and $u_{i}^{t}$ are related by the following equation:

$$
u_{i}^{t}(t)=u_{i}(t)+u_{g}(t)
$$

The displacements of the $n$ masses can be written as a matrix system as follows:

$$
\mathbf{u}^{t}(t)=\mathbf{u}(t)+\mathbf{1} u_{g}(t)=\mathbf{u}(t)+\mathbf{x}(t)
$$

$\mathbf{1}=\left(\begin{array}{lllll}1 & 1 & \cdots & 1 & 1\end{array}\right)^{T}$ is a $(n \times 1)$ vector where each component is one.

The dynamic equation for a base excitation is:

$$
\mathbf{f}_{I}+\mathbf{f}_{D}+\mathbf{f}_{S}=0
$$

where:

$$
\mathbf{f}_{I}=\mathbf{M} \ddot{\mathbf{u}}^{t}(t) ; \quad \mathbf{f}_{D}=\mathbf{B} \dot{\mathbf{u}}(t) ; \quad \mathbf{f}_{S}=\mathbf{K u}(t)
$$

expressing the fact that the inertia forces are related to the absolute acceleration $\ddot{\mathbf{u}}^{t}$, the damping forces $f_{D}$ to the relative velocity $\dot{\mathbf{u}}$, and the elastic forces $f_{S}$ to the relative displacement $u$ of the masses. Taking into account Eq. (18), the equation of motion (19a) can be written as:

$$
\mathbf{M} \ddot{\mathbf{u}}(t)+\mathbf{B} \dot{\mathbf{u}}(t)+\mathbf{K u}(t)=-M \ddot{\mathbf{x}}(t)
$$

where $\ddot{\mathbf{x}}(t)=\mathbf{1} \ddot{u}_{g}(t)=\mathbf{1} p(t)$ is the excitation at the base.

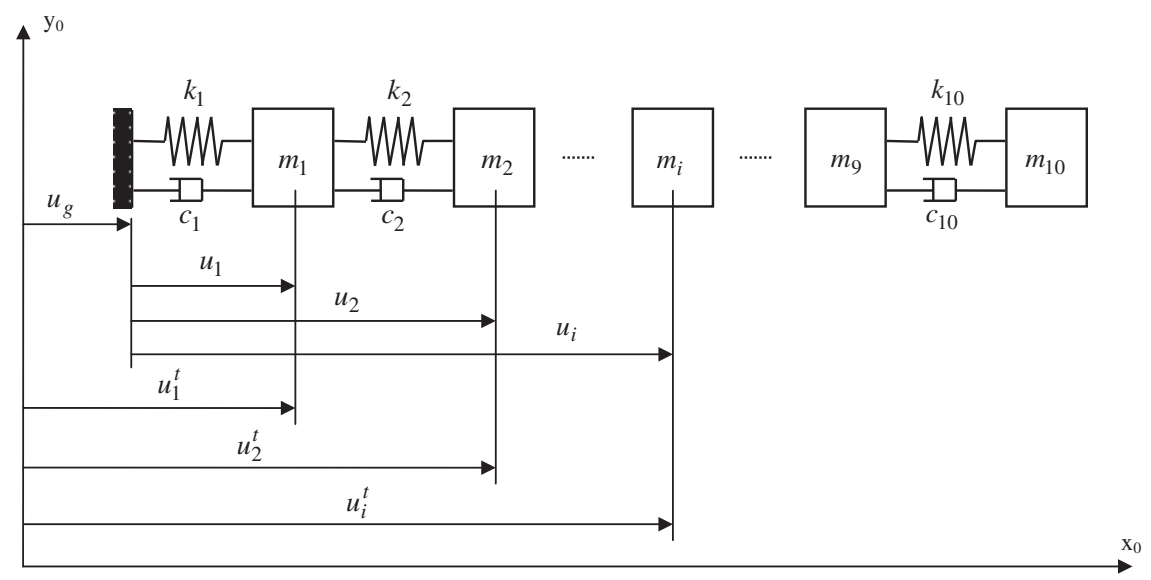

Fig. 2. Linear array of ten spring-mass oscillators. $m_{1}=\cdots=m_{10}=10 \mathrm{Mg}, k_{1}=k_{2}=k_{3}=40 \mathrm{MN} / \mathrm{m}, k_{4}=k_{5}=k_{6}=36 \mathrm{MN} / \mathrm{m}, k_{7}=k_{8}=k_{9}=k_{10}=32$ $M N / m, c_{i}=2 \xi_{i} \sqrt{m_{i} k_{i}}$ and $\xi_{i}=0.04$ for $i=1,2, \cdots, 10$. 
In this example, the system matrices defined by Jensen and Valdebenito [50] are

$$
\begin{aligned}
& \mathbf{M}=\left(\begin{array}{ccccc}
m_{1} & 0 & 0 & \ldots & 0 \\
0 & m_{2} & 0 & \ldots & 0 \\
\cdot & \cdot & \cdot & \ldots & \cdot \\
0 & \cdots & 0 & 0 & m_{10}
\end{array}\right) \\
& \mathbf{B}=\left(\begin{array}{ccccc}
c_{1}+c_{2} & -c_{2} & 0 & \cdots & 0 \\
-c_{2} & c_{2}+c_{3} & -c_{3} & \cdots & 0 \\
\cdot & \cdot & \cdot & \ldots & \cdot \\
0 & \cdots & 0 & -c_{10} & c_{10}
\end{array}\right) \\
& \mathbf{K}=\left(\begin{array}{ccccc}
k_{1}+k_{2} & -k_{2} & 0 & \ldots & 0 \\
-k_{2} & k_{2}+k_{3} & -k_{3} & \ldots & 0 \\
\cdot & \cdot & \cdot & \ldots & \cdot \\
0 & \ldots & 0 & -k_{10} & k_{10}
\end{array}\right)
\end{aligned}
$$

The random excitation $p(t)$ is modeled by a modulated filtered white noise:

$$
p(t)=\Omega_{1 g}^{2} v_{f 1}(t)+2 \zeta_{1 g} \Omega_{1 g} v_{f 2}(t)-\Omega_{2 g}^{2} v_{f 3}(t)-2 \zeta_{2 g} \Omega_{2 g} v_{f 4}(t)
$$

where $v_{f i}(t), i=1, \ldots, 4$ are functions in time, $\zeta_{\text {ig }}, i=1,2$ and $\Omega_{i g}, i=1,2$ are constant coefficients with

$$
\frac{d}{d t}\left(\begin{array}{c}
v_{f 1}(t) \\
v_{f 2}(t) \\
v_{f 3}(t) \\
v_{f 4}(t)
\end{array}\right)=\left(\begin{array}{cccc}
0 & 1 & 0 & 0 \\
-\Omega_{1 g}^{2} & -2 \zeta_{1 g} \Omega_{1 g} & 0 & 0 \\
0 & 0 & 0 & 1 \\
\Omega_{1 g}^{2} & 2 \zeta_{1 g} \Omega_{1 g} & -\Omega_{2 g}^{2} & -2 \zeta_{2 g} \Omega_{2 g}
\end{array}\right)\left(\begin{array}{c}
v_{f 1}(t) \\
v_{f 2}(t) \\
v_{f 3}(t) \\
v_{f 4}(t)
\end{array}\right)+\left(\begin{array}{c}
0 \\
\omega(t) \\
0 \\
0
\end{array}\right)
$$

In Eq. (25), $\omega(t)$ represents a Gaussian white noise with an autocorrelation function $E[\omega(t) \omega(t+\tau)]=I \delta(\tau) h^{2}(t)$, where $I$ denotes the intensity of the white noise, and

$$
h(t)= \begin{cases}0 & \text { for } t \leq 0 s \\ t / 2 & \text { for } 0 s \leq t \leq 2 s \\ 1 & \text { for } 2 s \leq t \leq 10 s \\ \exp (-0.1(t-10)) & \text { for } t \geq 10 s\end{cases}
$$

The values $\Omega_{1 g}=15.0 \mathrm{rad} / \mathrm{s}, \zeta_{1 g}=0.8, \Omega_{2 g}=0.3 \mathrm{rad} / \mathrm{s}, \zeta_{2 g}=0.995$ and white noise intensity $I=0.08 \mathrm{~m}^{2} / \mathrm{s}^{3}$ are used here to model the filter and independent normally distributed impulses are applied. The impulse of each discrete time step $t_{k}$, $k=1, \ldots, n_{T}$ has zero mean and standard deviation $h\left(t_{k}\right) \sqrt{I / \Delta t}$. The sampling interval and the duration of the excitation are taken as $\Delta t=0.005 \mathrm{~s}$ and $T=20 \mathrm{~s}$, respectively. Hence, the number of random variables that characterizes the uncertain excitation is equal to 4000 . Equivalently, the filter can be written in terms of the second-order differential equation

$$
\ddot{\mathbf{y}}(t)+\mathbf{B}_{f} \dot{\mathbf{y}}(t)+\mathbf{K}_{f} \mathbf{y}(t)=\mathbf{g}_{f} \omega(t)
$$

with

$$
\begin{aligned}
& \left.\mathbf{B}_{f}=\begin{array}{cc}
2 \zeta_{1 g} \Omega_{1 g} & 0 \\
-2 \zeta_{1 g} \Omega_{1 g} & 2 \zeta_{2 g} \Omega_{2 g}
\end{array}\right), \quad \mathbf{y}(t)=\left(\begin{array}{l}
v_{f 1}(t) \\
v_{f 3}(t)
\end{array}\right) \\
& \mathbf{K}_{f}=\left(\begin{array}{cc}
\Omega_{1 g}^{2} & 0 \\
-\Omega_{1 g}^{2} & \Omega_{2 g}^{2}
\end{array}\right), \quad \mathbf{g}_{f}=\left(\begin{array}{l}
1 \\
0
\end{array}\right)
\end{aligned}
$$

and

$$
p(t)=\mathbf{Q}_{y} \mathbf{y}(t)+\mathbf{Q}_{y} \dot{\mathbf{y}}(t),
$$

where

$$
\mathbf{Q}_{y}=\left(\begin{array}{ll}
\Omega_{1 g}^{2} & -\Omega_{2 g}^{2}
\end{array}\right), \quad \mathbf{Q}_{\dot{y}}=\left(2 \zeta_{1 g} \Omega_{1 g} \quad-2 \zeta_{2 g} \Omega_{2 g}\right)
$$

\subsubsection{Case of epistemic uncertainty}

We will consider the structure with epistemic uncertainty in the elements $m_{i}, c_{i}, k_{i}, i=1,2,3,8,9,10$ while the remainder of the structure is assumed to be deterministic. 
This scenario is devoted to the analysis of reliability based on a failure condition defined as a upper limit on the 10th eigenfrequency such that: $g=f_{10}-f_{\text {limit }}$.

Figs. 3 and 4 illustrate the evolution of the reliability and COV of probability of failure as a function of the dispersion levels.

In Figs. 3 and 4, the results of the IS method for 2000 samples are compared with those of the MCS method for 10,000 samples. The examination of these results shows that the IS method allows the prediction of the reference solution obtained using the MCS method.

The performances of the two sampling methods are given in Table 1. There is a reduction in the calculation time of $79.80 \%$ for IS, in comparison to the MCS reference method. This study supports the choice of the IS method as a robust tool for evaluating reliability.

In Fig. 3, the curves provide important information concerning the relative sensitivities of individual frequencies as a function of the level of uncertainty, in particular, how much uncertainty can be tolerated while satisfying a given level of performance.

\subsubsection{Case of hybrid uncertainty}

In this section, we combine the two types of uncertainties. In addition to the epistemic uncertainty in the structure ( $m_{i}$, $\left.c_{i}, k_{i}, i=1,2,3,8,9,10\right)$, we assume that the input is a random excitation $p(t)$.

The failure condition is defined by the maximum relative displacements between two consecutive DOF's over the time interval $[0 \mathrm{~s}, 20 \mathrm{~s}]$. In particular, four cases are considered here. Cases 1 and 2 correspond to the maximum relative

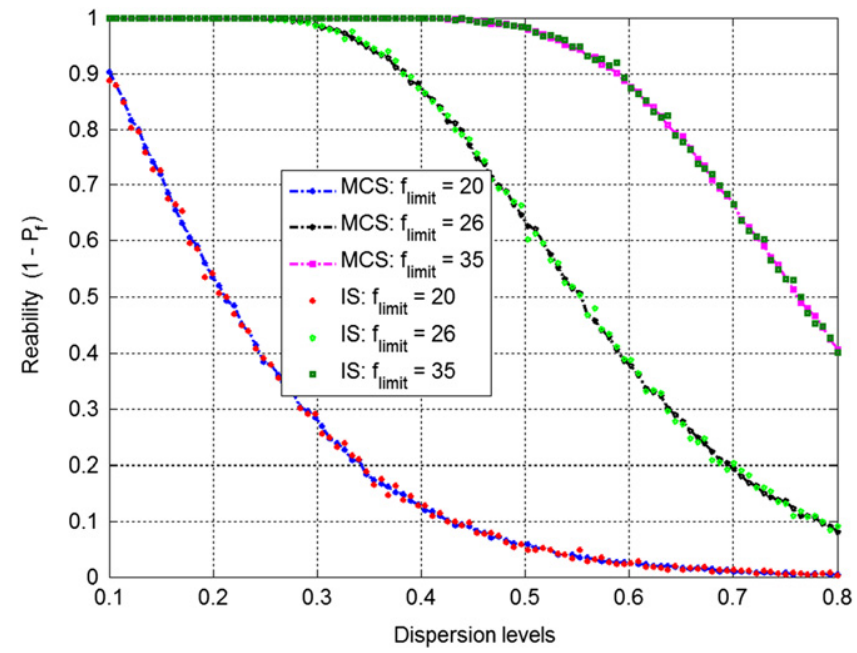

Fig. 3. Reliability according to the levels of dispersion, obtained by MCS (Number of simulations: 10,000) or IS (Number of simulations: 2000).

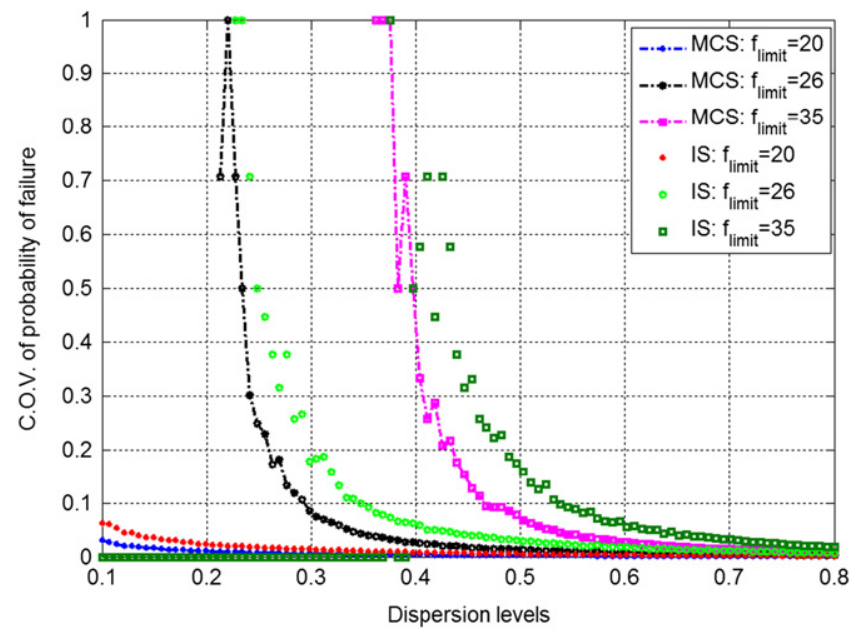

Fig. 4. COV of probability of failure according to the levels of dispersion, obtained by MCS or IS 
Table 1

Comparison time.

\begin{tabular}{lll}
\hline & MCS 10,000 runs & IS 2000 runs \\
\hline CPU Times (\%) & 100 & 20.20 \\
Reduction (\%) & & 79.80 \\
\hline
\end{tabular}

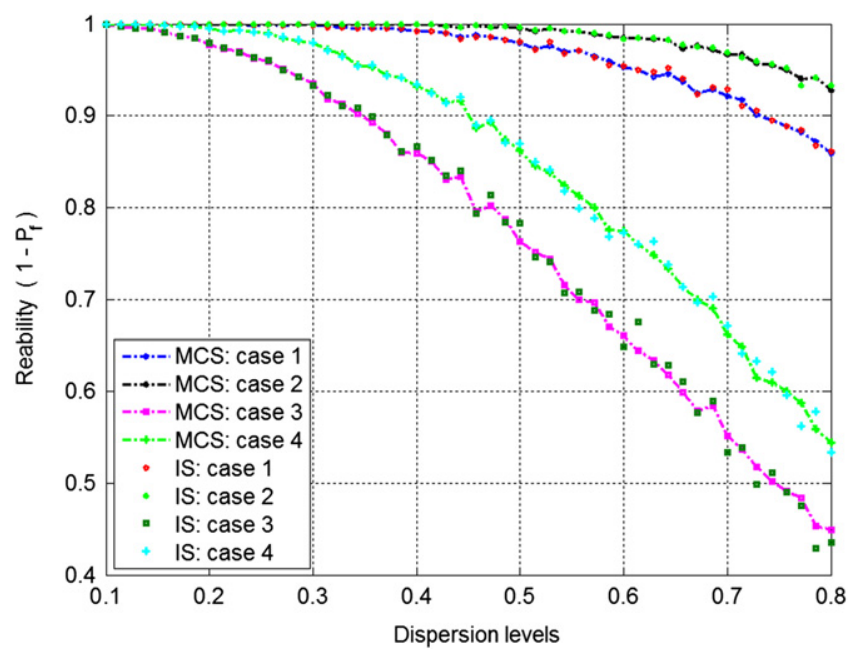

Fig. 5. Reliability according to the levels of dispersion, obtained by MCS (Number of simulations: 10,000) or IS (Number of simulations: 2000).

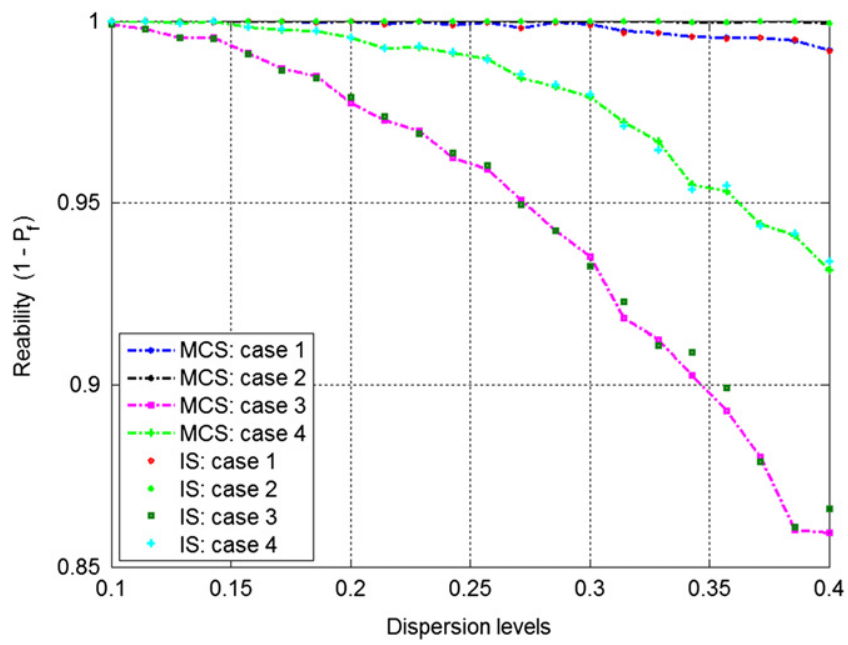

Fig. 6. Zoom of the reliability according to the levels of dispersion, obtained by MCS or IS.

displacement of the first degree of freedom for threshold levels equal to $0.057 \mathrm{~m}$ and $0.073 \mathrm{~m}$, respectively. Cases 3 and 4 correspond to the maximum relative displacement between the ninth and tenth degrees of freedom for threshold levels equal to $0.013 \mathrm{~m}$ and $0.017 \mathrm{~m}$, respectively.

Figs. 5 and 6 illustrate the evolution of the reliability as a function of the dispersion levels. Examination of the results shows that the IS method once again provides a good estimate of the reference solution obtained by MCS.

This result illustrates the impact of uncertainty in the dispersion parameter on the structural reliability. Indeed, for cases 1 and 2, the reliability analysis is seen to be robust, meaning relatively large epistemic uncertainty can be tolerated without a significant change in the probability of failure, while for cases 3 and 4 the reliability rapidly decreases starting at a dispersion level equal to 0.4 . 


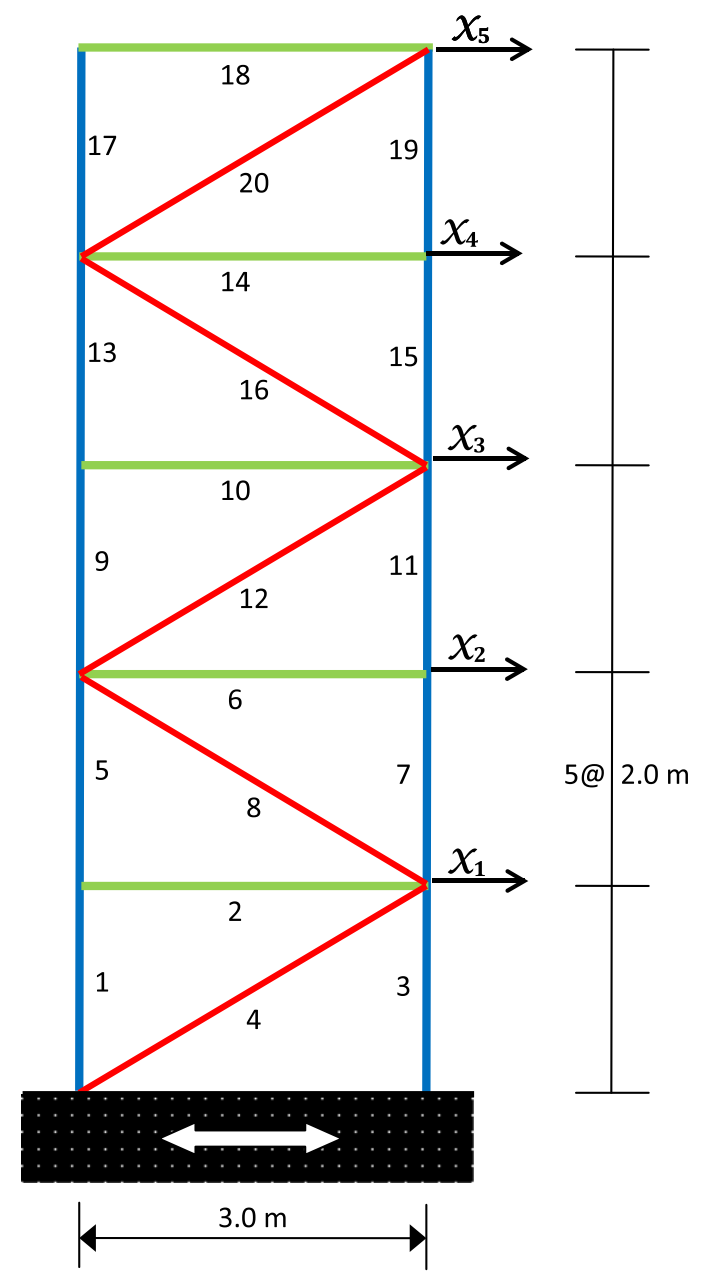

Fig. 7. Multistoried portal with random element properties.

\subsection{Frame structure}

We now consider a multistoried portal frame with 20 members. The details of the structure are shown in Fig. 7 with element numbering and node numbering. It is assumed that the axial stiffness (EA) and the bending stiffness (EI) of each member are Gaussian random variables so that there are in total 20 random variables, $x \in \mathfrak{R}^{20}$. As in the previous example it is also assumed that EA and EI of different members are uncorrelated. There are three types of elements and the properties of each element type are shown in Table 2. It is assumed that the column members are of type 1, the beam members are of type 2 and the bracing members are of type 3.

The random excitation is modeled by a modulated filtered white noise (Eq. (24)).

The failure condition is defined by the maximum relative displacements $\delta u_{\text {max }}$ between two consecutive floors (DOF's) over the time interval $[0.0 \mathrm{~s}, 20.0 \mathrm{~s}$ ]. The sampling interval is fixed to $0.01 \mathrm{~s}$. For numerical calculations it is assumed that $\delta u_{\max }=0.03 \mathrm{~m}$ for the 2nd floor $\left(X_{2}-X_{1}\right), \delta u_{\max }=0.035 \mathrm{~m}$ for the 3nd floor $\left(X_{3}-X_{2}\right), \delta u_{\max }=0.042 \mathrm{~m}$ for the $4 \mathrm{nd}$ floor $\left(X_{4}-X_{3}\right)$ and $\delta u_{\max }=0.052 \mathrm{~m}$ for 5 nd floor $\left(X_{5}-X_{4}\right)$.

Figs. 8 and 9 illustrate the evolution of the reliability as a function of the dispersion levels.

\section{Conclusions}

Model-based reliability analysis provides a means of quantifying the degree of confidence in the performance of a complex system. However, special attention must be given to studying the impact of assumptions concerning the way uncertainties are quantified on the analysis results. In this article, a strategy is proposed to investigate the robustness of a classical reliability analysis with respect to model uncertainty introduced using a non-parametric approach. The robustness of the reliability analysis is evaluated with respect to the degree of lack of knowledge in the associated dispersion parameters since these may be difficult to estimate in practice. If reasonable levels of lack of knowledge can be 
Table 2

Element types and associated elements numbers of the random multistoried portal frame.

\begin{tabular}{|c|c|c|c|c|c|}
\hline \multicolumn{6}{|c|}{ Epistemic uncertainty } \\
\hline \multirow[t]{2}{*}{ Element type } & \multicolumn{2}{|l|}{ EA (N) } & \multicolumn{2}{|l|}{ EI $\left(\mathrm{Nm}^{2}\right)$} & \multirow[t]{2}{*}{ Element numbers } \\
\hline & mean & $\delta_{G}$ & mean & $\delta_{G}$ & \\
\hline 1 & $5.0 \times 10^{12}$ & $0.1-0.8$ & $6.0 \times 10^{7}$ & $0.1-0.8$ & $1,3,5,7,9,11,13,15,17,19$ \\
\hline \multicolumn{6}{|c|}{ Parametric uncertainty } \\
\hline \multirow[t]{2}{*}{ Element type } & \multicolumn{2}{|l|}{ EA (N) } & \multicolumn{2}{|l|}{ EI $\left(\mathrm{Nm}^{2}\right)$} & \multirow[t]{2}{*}{ Element numbers } \\
\hline & mean $\left(\times 10^{12}\right)$ & $\operatorname{COV}(\%)$ & mean $\left(\times 10^{7}\right)$ & $\operatorname{cov}(\%)$ & \\
\hline 2 & 3.0 & 3.0 & 4.0 & 10.0 & $2,6,10,14,18$ \\
\hline 3 & 1.0 & 10.0 & 2.0 & 9.0 & $4,8,12,16,20$ \\
\hline
\end{tabular}

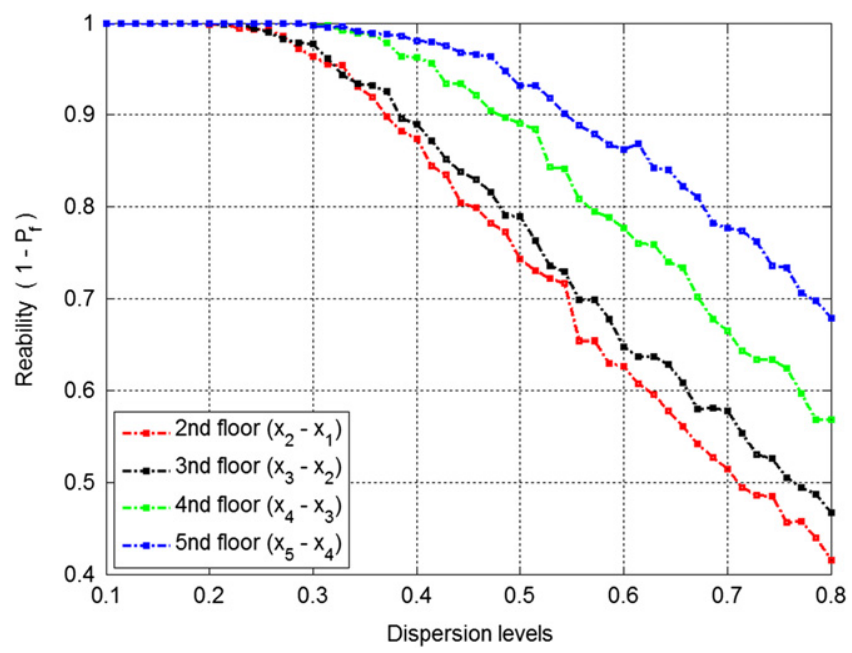

Fig. 8. Reliability according to the levels of dispersion, obtained by IS (Number of simulations: 1000).

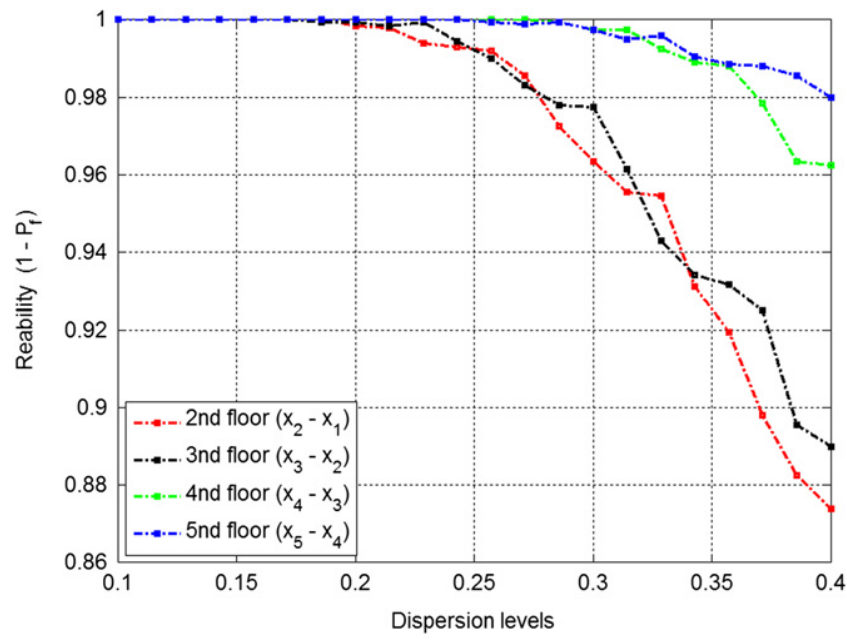

Fig. 9. Zoom of the Reliability according to the levels of dispersion.

tolerated while still providing acceptable reliability, then the credibility in the design and analysis is accrued. Academic examples are used to illustrate the proposed strategy and an importance sampling method is used in the reliability analysis in order to reduce calculation time. 


\section{References}

[1] V. Plevris, Innovative Computational Techniques for the Optimum Structural Design Considering Uncertainties, Ph.D. Dissertation, 2010, Institute of Structural Analysis and Seismic Research, Athens.

[2] G.I. Schuëller, Special issue on computational methods in stochastic mechanics and reliability analysis, Comput. Meth. Appl. Mech. Eng. 194 (12-16) (2005) 1251-1795

[3] Y. Tsompanakis, N.D. Lagaros, M. Papadrakakis, Structural optimization considering uncertainties, in: D.M. Frangopol (Ed.), Structures and Infrastructures, Taylor \& Francis, 2008.

[4] G.I. Schuëller, H.A. Jensen, Computational methods in optimization considering uncertainties-an overview, Comput. Meth. Appl. Mech. Eng. 198 (2008) 2-13.

[5] W.L. Oberkampf, S.M. DeLand, B.M. Rutherford, K.V. Diegert, K.F. Alvin, Estimation of Total Uncertainty in Modeling and Simulation, Sandia Report SAND2000-0824, Albuquerque, NM 2000.

[6] L.-P. He, H.-Z. Huang, L. Du, X.-D. Zhang, Q. Miao, A Review of Possibilistic Approaches to Reliability Analysis and Optimization in Engineering Design, Human-Computer Interaction, Part IV, HCII 2007, LNCS 4553, Springer-Verlag, Berlin Heidelberg, 2007, pp. 1075-1084.

[7] A. Der Kiureghian, O. Ditlevsen, Aleatory or epistemic? Does it matter? Struct. Saf. 31 (2) (2009) 105-112.

[8] F. Tonon, A. Bernardini, I. Elishakoff, Hybrid analysis of uncertainty: probability, fuzziness and anti-optimization, Chaos, Solitons Fract. 12 (2001) $1403-1414$

[9] O. Rollot, I. Elishakoff, Large variation finite element method for beams with stochastic stiffness, Chaos, Solitons Fract. 17 (2003) $749-779$.

[10] B. Goller, H.J. Pradlwarter, G.I. Schuëller, Robust model updating with insufficient data, Comput. Meth. Appl. Mech. Eng. 198 (2009) 3096-3104.

[11] J. Jakeman, M. Eldred, D. Xiu, Numerical approach for quantification of epistemic uncertainty, J. Computat. Phys. 229 (2010) 4648-4663.

[12] E.T. Jaynes, Information theory and statistical mechanics, Phys. Rev. 108 (2) (1957) 171-190.

[13] J.N. Kapur, H.K. Kesavan, Entropy Optimization Principles with Applications, Academic Press, San Diego, New York, 1992.

[14] C. Soize, A nonparametric model of random uncertainties for reduced matrix models in structural dynamics, Probabilist. Eng. Mech. 15 (3) (2000) $277-294$.

[15] S. Adhikari, A non-parametric approach for uncertainty quantification in elastodynamics, in: 47th AIAA/ASME/ASCE/AHS/ASC Structures, Structural Dynamics \& Materials Conference, Newport, Rhode Island, USA, May 2006.

[16] S. Adhikari, An unified parametric-nonparametric uncertainty quantification approach for linear dynamical systems, in: 48th AIAA/ASME/ASCE/AHS/ ASC Structures, Structural Dynamics \& Materials Conference, 23-26 April 2007, Waikiki, Hawaii.

[17] G.J. Klir, R.M. Smith, On measuring uncertainty and uncertainty-based information: Recent developments, Ann. Math. Artif. Intel. 32 (2001) 5-33.

[18] S. Adhikari, K. Lonkar, M.I. Friswell, Experimental case studies on uncertainty quantification in structural dynamics, in: Proceedings of the 25th International Modal Analysis Conference (IMAC-XXV), Orlando, Florida, USA, February 2007.

[19] Y. Ben-Haim, I. Elishakoff, Convex Models of Uncertainty In Applied Mechanics, Elsevier Science, Amsterdam, 1990.

[20] Y. Ben-Haim, A non-probabilistic measure of reliability of linear systems based on expansion of convex models, Struct. Saf. 17 (1995) 91-109.

[21] Y. Ben-Haim, Info-gap decision theory: decisions under serve uncertainty, Academic Press, New York, 2001.

[22] I. Elishakoff, Discussion on: A non-probabilistic concept of reliability, Struct. Saf. 17 (3) (1995) 195-199.

[23] F. Au, Y.S. Cheng, L.G. Tham, G.W. Zeng, Robust design of structures using convex models, Comput. Struct. 81 (2003) 2611-2619.

[24] Y. Luo, Z. Kang, A. Li, Structural reliability assessment based on probability and convex set mixed model, Comput. Struct. 87 (2009) 1408-1415.

[25] Z. Ni, Z. Qiu, Hybrid probabilistic fuzzy and non-probabilistic model of structural reliability, Comput. Ind. Eng. 58 (2010) $463-467$.

[26] I. Elishakoff, P. Colombi, Combination of probabilistic and convex models of uncertainty when scarce knowledge is present on acoustic excitation parameters, Comput. Meth. Appl. Mech. Eng. 104 (2) (1993) 187-209.

[27] W.L. Oberkampf, J.C. Helton, C.A. Joslyn, S.F. Wojtkiewicz, Challenge problems: uncertainty in system response given uncertain parameters, Reliab. Eng. Syst. Saf. $85(1-3)(2004) 11-19$.

[28] R.C. Penmetsa, R.V. Grandhi, Efficient estimation of structural reliability for problems with uncertain intervals, Comput. Struct. 80 (2002) $1103-1112$.

[29] J.W. Hall, J. Lawry, Generation combination and extension of random set approximations to coherent lower and upper probabilities, Reliab. Eng. Syst. Saf. 85 (2004) 89-101.

[30] D.R. Karanki, H.S. Kushwaha, A.K. Verma, S. Ajit, Uncertainty analysis based on probability bounds (p-box) approach in probabilistic safety assessment, Risk Anal. 29 (5) (2009) 662-675.

[31] Z. Kang, Y. Luo, Reliability-based structural optimization with probability and convex set hybrid models, Struct. Multidiscip. 042 (2010) 89-102.

[32] O.D. Ditlevsen, H.O. Madsen, Structural Reliability Method, John Wiley \& Sons, New York, 1996.

[33] X. Wang, Z. Qiu, I. Elishakoff, Non-probabilistic set-theoretic model for structural safety measure, Acta Mech. 198 (2008) 51-64.

[34] N. Rabhi, M. Guedri, H. Hassis, N. Bouhaddi, Structure dynamic reliability: A hybrid approach and robust meta-models, Mech. Syst. Sig. Process. 25 (2011) 2313-2323.

[35] P. Thoft-Christensen, M.J. Baker, Structural Reliability Theory and its Applications, Springer, Berlin, 1982.

[36] A. Chateauneuf, Principles of reliabilitybased design optimization, in: Y. Tsompanakis, N.D. Lagaros, M. Papadrakakis (Eds.), Structural Design Optimization Considering Uncertainties, Taylor \& Francis, 2008, pp. 3-30.

[37] B.M. Ayyub, C.Y. Chia, Generalised conditional expectation for structural reliability assessment, Struct. Saf. 11 (1992) $131-146$.

[38] M.R. Rajashekhar, B.R. Ellingwood, A new look at the response surface approach for reliability analysis, Struct. Saf. 12 (1993) 205-220.

[39] A. Florian, An efficient sampling scheme: updated Latin hypercube sampling, Probabilist. Eng. Mech. 7 (2) (1992) 123-130.

[40] C.G. Bucher, Adaptive sampling - an iterative fast Monte Carlo procedure, Struct. Saf. 5 (1988) 119-126.

[41] Y. Zheng, Y. Fujimoto, M. Iwata, An empirical fitting_adaptive approach to importance sampling in reliability analysis, J. Soc. Naval. Arch. Japan 171 (1991) 433-441.

[42] R. Srinivasan, Importance Sampling: Applications in Communications and Detection, SpringerVerlag, Berlin, 2002.

[43] P. Bjerager, Probability integration by directional simulation, J. Eng. Mech., ASCE 114 (8) (1988) 1285-1302.

[44] W.A. Gray, R.E. Melchers, Modifications to the 'directional simulation in the load space' approach to structural reliability analysis, Probabilist. Eng. Mech. 21 (2) (2006) 148-158.

[45] R.G. Ghanem, P.D. Spanos, Stochastic Finite Elements_A Spectral Approach, Spring, Berlin, 1991.

[46] C. Soize, Maximum entropy approach for modeling random uncertainties in transient elastodynamics, J. Acoust. Soc. Am. 109 (5) (2001) $1979-1996$.

[47] C. Soize, Random matrix theory for modeling uncertainties in computational mechanics, Comput. Meth. Appl. Mech. Eng. 194 (12-16) (2005) $1333-1366$

[48] M. Schinozuka, Monte Carlo Solution of structural dynamics, Comput. Struct. 2 (1972) 855-874.

[49] M. Marques, Reliability methods for passive systems, RMPS project, in: 5th Euratom Framework Programme 1998-2002.

[50] H.A. Jensen, M.A. Valdebenito, Reliability analysis of linear dynamical systems using approximate representations of performance functions, Struct. Saf. 29 (2007) 222-237.

[51] P. Paultre, Dynamiques des structures : application aux ouvrages de génie civil, Ed. Hermès, 2004. 